\title{
Comment on 'Diagnosis of pathological complete response to neoadjuvant chemotherapy in breast cancer by minimal invasive biopsy techniques'
}

Henry M Kuerer ${ }^{*}, 1$, Wei T Yang ${ }^{2}$ and Savitri Krishnamurthy ${ }^{3}$

${ }^{1}$ Department of Breast Surgical Oncology, The University of Texas MD Anderson Cancer Center, 1515 Holcombe Blvd., Houston, TX 77030, USA;

${ }^{2}$ Department of Diagnostic Radiology, The University of Texas MD Anderson Cancer Center, 1515 Holcombe Blvd., Houston, TX 77030, USA and

${ }^{3}$ Department of Pathology, The University of Texas MD Anderson Cancer Center, 1515 Holcombe Blvd., Houston, TX 77030, USA

Sir,

We congratulate the authors on performing this multicentre trial with 164 patients (Heil et al, 2015). One important selection criterion is the initial size of the primary tumor (T size) on imaging - this information did not appear in the manuscript and is of interest. The probability of adequately sampling the initial region of disease by image guidance is likely related to the $T$ size at diagnosis and the size of residual disease on imaging. Do the authors have information on the initial $\mathrm{T}$ stage of all patients and the size of residual imaging abnormality at the time of pre-surgical biopsy? This is not the first report regarding the concept of minimally invasive techniques for sampling to assess pathologic complete response in breast cancer (Clouth et al, 2007). The success of moving these studies into the realm of clinical omission of surgery is dependent on exquisite radiologic targeting of the lesion (37\% did not have a clip placed at initial biopsy in the current study), adequate sampling with vacuum-assisted biopsy (utilized in the current study in $\sim 30 \%$ ), and the number of biopsies performed (unknown $55 \%$ of cases). At MD Anderson, we have an actively accruing trial using image-guided vacuumassisted biopsy with fine-needle aspiration and more stringent selection criteria including patients with a $50-70 \%$ chance of pathologic eradication of disease after neoadjuvant chemo- and targeted therapy (triple-negative and HER2-amplified breast cancers) (National Institute of Health, 2015).

\section{CONFLICT OF INTEREST}

The authors declare no conflict of interest.

\section{REFERENCES}

Clouth B, Chandrasekharan S, Inwang R, Smith S, Davidson N, Sauven P (2007) The surgical management of patients who achieve a complete pathological response after primary chemotherapy for locally advanced breast cancer. Eur J Surg Oncol 33(8): 961-966.

Heil J, Kümmel S, Schaefgen B, Paepke S, Thomssen C, Rauch G, Ataseven B, Große R, Dreesmann V, Kühn T, Loibl S, Blohmer JU, von Minckwitz G (2015) Diagnosis of pathological complete response to neoadjuvant chemotherapy in breast cancer by minimal invasive biopsy techniques. Br J Cancer 113(11): 1565-1570.

National Institute of Health (2015) Feasibility and Identification of Breast Cancer Patients for Potential Avoidance of Surgery: Accuracy of image guided percutaneous sampling compared with surgery to evaluate eradication of breast cancer after preoperative chemotherapy. Available from https://clinicaltrials.gov/show/ NCT02455791.

*Correspondence: Dr HM Kuerer; E-mail: hkuerer@mdanderson.org

Published online 19 April 2016 